\title{
The Influence of Retinal Adaptation and Location on the "Empfindungszeit."
}

By

\author{
Tosisada Mita, Kazuo Hironaka and Izumi Koike. \\ （三田俊定） (弘中一焳) (小池泉)
}

(From the Physiological Laboratory of Prof. K. Motokawa, Tohoku University, Sendai.)

(Received for publication, September 5, 1949)

INTRODUCTION.

Fröhlich" used the term "Empfindungszeit" for the time which elapses between a stimulus and the sensation induced. The determinations of the "Empfindungszeit" ("E.Z.") were made by many investigators under controlled conditionș. Their values, however, depend on a variety of conditions, of which the most important are the retinal location and the adaptation of the eyes. From the different anatomical distribution of cones and rods in th retina and the functional differences between the fovea and the periphery, the characteristic differences may be supposed in the behaviour of the "E.Z." V. Kries") concluded, on the basis of the experiment performed by Engelking and Poos with Pulflich's method, that the "E.Z." for the twilight apparatus is longer than that for the daylight apparatus. On the other hand, Fröhlich ${ }^{11}$ and his co-workers ${ }^{3)}$ made objection to this conclusion. Thus, the problem is not yet settled.

We have two methods for measuring the absolute value of the "E.Z.", that is, Fröhlich's and Hazelhoff's method, so called from the authors' names. Some modifications to Hazelhoff's method ${ }^{4}$ were necessary for the present experiment. The modified method enabled us to measure the "E.Z." at any region of the retina.

\section{EXPERIMENTAL.}

Apparatus and Method.

Fig. 1 shows the arrangement of the apparatus diagrammatically. A circular disc (D), $57 \mathrm{~cm}$. in diameter, was driven by an electric motor, and the speed of the rotating disc was measured precisely by means of a stroboscope (St).

The disc was equipped with two red point lights $\left(f_{1}\right.$ and $\left.f_{2}\right)$, placed near the margin on a radius of the disc, $4.5 \mathrm{~cm}$. apart, subtending $1.5^{\circ}$ in visual angle, and these lights served as two fixation points. Their bright- 


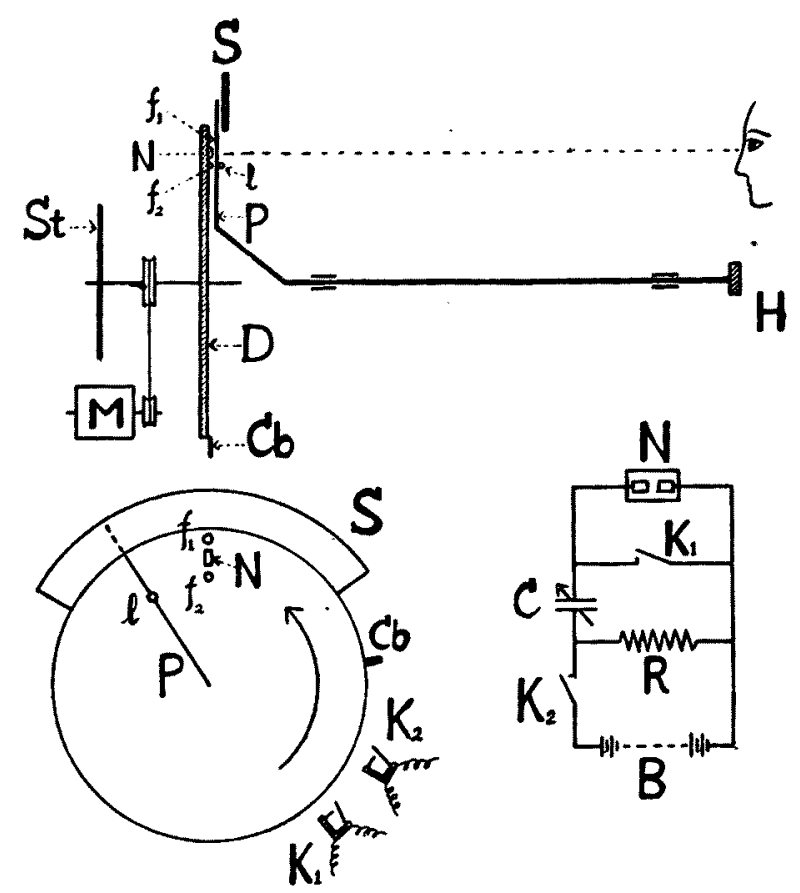

Fig. 1. Diagram of the arrangement for measuring the "E.Z.", B: 180 V. Battery, C: Variable condenser, Cb: Contact-breaker, D : Revolving disc, $\mathrm{f}_{1}$ and $\mathrm{f}_{2}$ : Fixation Points, $\mathrm{H}$ : Handle of pointer, $\mathrm{K}_{1}$ and $\mathrm{K}_{2}$ : Contact keys, 1 : Point light on the pointer, $\mathrm{M}$ : Electric motor, N: Neon tube, P: Pointer, R: Resistance, S: Scale, St: Stroboscope.

ness was controlled separately by two rheostats.

The stimulating light was the negative glow of a tiny discharge neon tube $(\mathrm{N})$, attached to the revolving disc; this neon tube could be placed in any desired position on the surface of the disc. The neon glow flashed on a condenser discharge which was started by opening two contact keys $\left(\mathrm{K}_{1}\right.$ and $\mathrm{K}_{2}$ ) one after the other by a contact breaker (CB) attached to the margin of the revolving disc. Corresponding to the intensity of the discharge current, the glow began with the maximum intensity of about 0.02$0.03 \mathrm{Hk} / \mathrm{cm}^{2}$, and declined rapidly. The duration of the glow was controlled by a variable condenser and was experimentally measured with a phosphoroscopic method. The illuminating patch consisting of the neon glow was rectangular, $2 \mathrm{~mm} . \times 3 \mathrm{~mm}$. in size, subtending $4^{\prime} \times 6^{\prime}$ in visual angle. When low brightness of the stimulus was necessary, the illuminating patch was covered with a number of calibrated film filters:

A pointer $(\mathrm{P})$ carrying a red point light $(l)$ was arranged coaxially with the revolving disc, and, operated manually by the observer, it was used to indicate the position of the flash coming in sight. The position indicated 
by the pointer was read on a scale by the experimenter. In experiments in the dark some fixed reference points were used so as to get better orientation of the flash. For this purpose two such weak red point lights which had no influence on the value of the "E.Z." were used. In experiments with light-adapted eyes three incandescent lamps were used for illumination of the surrounding field; the intensity of the illumination on the surface of the revolving disc ranged from 0.1 to 10,000 luxs and the brightness of the whole field was kept as uniform as possible.

The observer sat at a distance of $170 \mathrm{~cm}$. in front of the revolving disc and kept his head upright with the chin and forehead holders. When he received a light stimulus with the resting eyes, he, of course, saw it in the real direction, from which the light came. However, when he made his gaze follow the middle point of the two fixation points travelling at a constant speed, the direction towards which the perception was projected, did not coincide with the real direction, but showed some deflection towards the direction of the eye movement. Taking the reading of the deflection, the "E.Z." is defined as the amount of deflection divided by the velocity of the eye movement. When the fovea was tested, the illuminating patch was placed midway between two fixation points $\left(f_{1}\right.$ and $\left.f_{2}\right)$, and when the parafovea was tested, the patch was so adjusted that the image of the patch fell on the parafovea. In the above measurements, the illuminating patch was always moving at a constant speed, but for the measurement of the "E.Z." for the periphery the patch was fixed outside the revolving disc. The illuminating light was flashed at the moment when the moving fixation point passed a given point. In the latter two cases the observer had to indicate the position of the moving fixation point at the moment when he' saw the light at the parafovea or the periphery.

Measurements with dark-adapted eyes were made after a stay of at least 40 minutes in the dark. Measurements with light-adapted eyes were commenced after light adaptation of more than 15 minutes preceded by sufficient dark adaptation. Three subjects were employed for the measurement, of whom two had slightly corrected myopia. Data were the average of 10 readings taken at intervals of about 20 seconds.

\section{Results.}

\section{The eye movement and the "E.Z."}

Uniformity of the eye movement and the coincidence of the visual line of the observer with the fixation point travelling at a constant speed are the indispensable conditions of our method. In order to see whether these conditions were fulfilled in our experiments, the following experiment was made. Under a stationary light adaptation, the fovea is more sensitive 
than the parafovea. Therefore, the intensity of a stimulus can be so chosen as to be a little higher than the threshold intensity of the fovea, but lower than that of the parafovea. Then, if the eyes could not correctly follow. the travelling fixation point, the light of this intensity would not be seen because the light would fall outside the fovea. In fact, the light was always clearly perceived in this experiment. This fact indicates that the above conditions are satisfied in our experiments.

The "E.Z." for the fovea was now measured binocularly with various speeds of the fixation point ranging from $10^{\circ}$ up to $44^{\circ}$ per sec. in visual angle to study the effect of the speed on the "E.Z." In Table I, two examples are shown which indicate that the speed has no effect on the "E.Z." for the fovea. We therefore usually employed the most suitable speed $\left(22^{\circ}\right.$ per sec. in visual angle) throughout the experiment.

\section{TABLE I.}

Relation of "E.Z." to Speed of Eye Movement

Light stimulus: brightness approximately $0.02 \mathrm{c} . / \mathrm{cm}^{2}$, duration $1,1 \mathrm{msec}$, area $6^{\prime} \times 4^{\prime}$ in visual angle. Binocular vision. Subject T. M.

\begin{tabular}{l|rrrrrrrr}
\hline & \multicolumn{7}{|c}{ Speed of Eye Movernent: (degrees in visual angle)/sec. } \\
& $10.0^{\circ}$ & $14.7^{\circ}$ & $18.9^{\circ}$ & $22.0^{\circ}$ & $26.4^{\circ}$ & $33.0^{\circ}$ & $37.7^{\circ}$ & $44.0^{\circ}$ \\
\cline { 1 - 9 } "E.Z." and probable error in & 66.7 & 69.0 & 66.9 & 63.4 & 64.6 & 73.3 & 68.0 & 78.4 \\
msec. (dark adaptation) & \pm 6.5 & \pm 7.0 & \pm 10.1 & \pm 9.6 & \pm 8.0 & \pm 3.3 & \pm 4.1 & \pm 2.7 \\
\hline "E.Z." and probable error in & 111.9 & 118.2 & 118.2 & 113.3 & 115.0 & 108.0 & 105.3 & 96.0 \\
msec. (light adaptation to 10 & \pm 14.1 & \pm 14.3 & \pm 16.1 & \pm 12.8 & \pm 6.7 & \pm 3.3 & \pm 4.1 & \pm 3.9
\end{tabular}

2. "E.Z." for the fovea and the parafovea.

A series of measurements of the "E.Z." for the fovea and the parafovea was made binocularly at various levels of adaptation. Two different intensities of light stimuli were employed, of which the higher one was far above the threshold intensity for the fovea and it was taken as unity, while the lower one was $1 / 71$ unit corresponding approximately to the threshold intensity of the fovea of the completely dark-adapted eyes. Results are shown in Fig. 2. In a given state of adaptation, the " E.Z." obtained with 1 unit intensity of the stimulus showed the shortest value for the fovea, and those for the parafovea increased with increasing eccentricity from the center of the retina (see curves $\mathrm{A}, \mathrm{B}, \mathrm{C}, \mathrm{D}$, and $\mathrm{E}$ ). This behaviour of the "E.Z." could be seen in every state of adaptation. The "E.Z." for each position increased with progress of light adaptation; a curve of the " $E$. Z.'s" plotted as ordinates against degrees eccentric from the fovea as abscissæ shifted upwards with light adaptation. But the upward shifting 


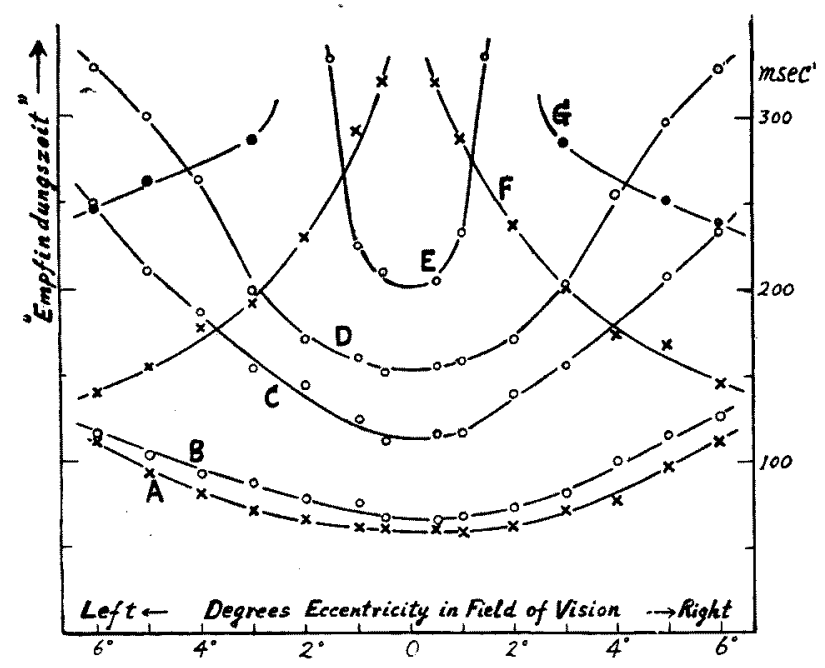

Fig. 2. "E.Z." for fovea and parafovea.

Curves A, B, F, and G were obtained under dark adaptation, C, D and E under light adaptation to 1 lux, 100 luxs and 10,000 luxs respectively. Intensity of the light stimulus is 1 unit for curves A, B, C, D and E, 1/71 unit for $F$ and $G$. Duration of light is $14.5 \mathrm{msec}$. for curves $B, C, D$ and $E, 1.7 \mathrm{msec}$. for A and F, $1.3 \mathrm{msec}$. for G. Subject is T. M. for curves B, C, D, E and G, K. H. for $A$ and $F$.

of the curve was not uniform for every area tested: the more eccentric the location, the longer was the "E.Z." In order to see this relation more clearly, the ratio of the "E.Z." for each parafoveal region to that for the fovea was calculated, as shown in Table II. Those ration for any retinal

\section{T A B LE II.}

Ratios of "E.Z." for each Region to that for Fovea.

Binocular vision. Light stimulus: brightness approximately $0.02 \mathrm{c} . / \mathrm{cm}^{2}$, duration $14.5 \mathrm{msec}$, area $6^{\prime} \times 4^{\prime}$ in visual ängle. Subject T. M.

\begin{tabular}{|c|c|c|c|c|c|c|c|c|}
\hline & & \multicolumn{7}{|c|}{ Degrees of eccentricity in field of vision } \\
\hline & & $0.5^{\circ}$ & $1.0^{\circ}$ & $2.0^{\circ}$ & $3.0^{\circ}$ & $4.0^{\circ}$ & $5.0^{\circ}$ & $6.0^{\circ}$ \\
\hline \multirow[t]{2}{*}{ Dark adaptation } & Right field & 1.00 & 1.05 & 1.12 & 1.24 & 1.53 & 1.76 & 1.91 \\
\hline & Left field & 1.03 & 1.17 & 1.20 & 1.33 & 1.52 & 1.58 & 1.77 \\
\hline \multirow{2}{*}{$\begin{array}{l}\text { Light adaptation } \\
\text { to } 1 \text { lux }\end{array}$} & Right field & 1.04 & 1.04 & 1.25 & 1.40 & 1.59 & 1.86 & 2.09 \\
\hline & Left field & 1.00 & 1.11 & 1.29 & 1.38 & 1.67 & 1.89 & 2.23 \\
\hline \multirow{3}{*}{$\begin{array}{l}\text { Light adaptation } \\
\text { to } 100 \text { luxs }\end{array}$} & Right field & 1.00 & 1.06 & 1.13 & 1.31 & 1.73 & 1.98 & 2.16 \\
\hline & Left field & 1.03 & 1.04 & 1.13 & 1.34 & 1.68 & 1.96 & 2.16 \\
\hline & mean & 1.02 & 1.08 & 1.19 & 1.33 & 1.62 & 1.84 & 2.05 \\
\hline
\end{tabular}


region showed approximately a constant value in all three states of adaptation, and they increased with increasing eccentricity from the center of the retina.

The fact that the "E.Z." for a constant intensity of light increases with progress of light adaptation by the eyes may be deduced from the photochemical theory of vision that the reduction of the photosensitive substances due to light adaptation causes the retardation of the subsequent reaction in the retina. But the regional difference of the "E.Z." under a given adaptation will have a bearing on the other factors, not only these such as intensities and wave-lengths of light but also on the regional difference of the anatomical structure of the retina. When the intensity of the stimulus was reduced below the threshold intensity of the fovea, the result was reversed to the former one. Curves $F$ and $G$ in Fig. 2 represent data of measurements with a light stimulus of $1 / 71$ unit under dark adaptation. The "E.Z." showed the largest value at the center and it decreased with increasing eccentricity from the center, as was expected from the density distribution of the rod in the retina. Thus, the slope of curves are quite reverse to that of curves $\mathrm{A}, \mathrm{B}, \mathrm{C}, \mathrm{D}$ and $\mathrm{E}$ in the above experiment. This difference is probably due to the fact that the "E.Z." is determined predominantly by the rod function in this case, while in the former case it is determined by the cone function.

\section{3. "E.Z." for the periphery.}

A series of measurements of the "E.Z." for the periphery was made uniocularly with three different intensities of light for regions ranging from $2^{\circ}$ to $90^{\circ}$ on the nasal horizontal meridian of the right eye, completely darkadapted. Fig. 3 shows four examples. Tracing the curves of Fig. 3 from the center towards the periphery, we see that the curves for 1 unit intensity of light rises at first, reaches a maximum at about $10^{\circ}$, falls again to a minimum at about $30^{\circ}$ and rises gradually towards the periphery. The other three curves B for $1 / 71$ unit, $\mathrm{C}$ and $\mathrm{D}$ for $1 / 5000$ unit are different in their courses from the curve A. They fall from the beginning, reach a minimum at about $30^{\circ}$ and then rise again towards the periphery. Above all, it is a most remarkable fact that both curves $A$ and $B$ overlap the one above the other within a range between about $10^{\circ}$ and $50^{\circ}$.

\section{Discussion.}

As slight adaptation causes an increase in "E.Z." and makes it difficult to determine the "E.Z." for the periphery, dark adaptation is preferable to light adaptation for a comparison of both "E.Z.'s" determined by cones and rods. 


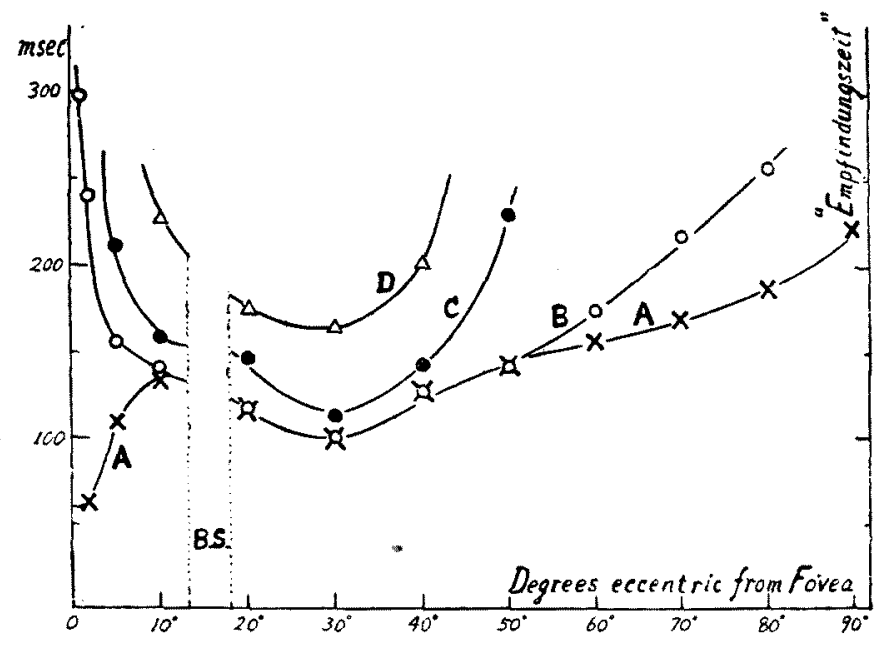

Fig. 3. "E.Z." for periphery on nasal horizontal meridian of the darkadapted right eye.- Intensity of light is 1 unit for curve $A, 1 / 71$ for $B, 1 / 5000$ for $\mathrm{C}$ and D. Duration of light is $1.3 \mathrm{msec}$. for $\mathrm{A}$ and $\mathrm{B}, 18.5 \mathrm{msec}$. for $\mathrm{C}$ and $9.5 \mathrm{msec}$. for D. Subject is K. H. for curvers $A$ and $B, T$. M. for $C$ and $D$.

If we assume that such weak light as corresponds to the subliminal intensity of the fovea of the dark-adapted eyes stimulates merely the rods in the retina but not the cones, the "E.Z." measured with such intensities must concern only the rod function. Then, from the inspection of the curves $B, C$ and D, in Fig. 3 the density of the stimulated rods is considered to be a determining factor of the "E.Z."; the larger the number of the rods stimulated, the shorter the "E.Z."

On the other hand, the curve A in Fig. 3 which was obtained with an intensity of light sufficiently high to stimulate the cones, shows a different behaviour from the curve B in Fig. 3 obtained with a low intensity; this difference may by due to the participation of the cones. Comparing the curves $A$ and $B$, we find that increases in intensity shorten the "E.Z.'s" for the parafovea and the extreme periphery, but have no effect on the "E.Z.'s" for the middle periphery from $10^{\circ}$ to $50^{\circ}$, namely that the curve $\mathrm{A}$ is divided into three functionally different parts.

Osterberg ${ }^{51}$ reported reliable data on the density distribution of cones and rods in the human retina. The density of rods in the nasal horizontal meridian of the retina takes a maximum value at $22^{\circ}$ and decreases gradually on either side of this region, while the density of the cones on the same meridian decreases with increasing eccentricity from the center at first rapidly then gradually, reaches approximately a constant value at about $10^{\circ}$ and keeps almost the same value within the wide range between $10^{\circ}$ and $80^{\circ}$. 
It is noted that the density of cones is about the same at $10^{\circ}$ and $50^{\circ}$, and that the same is the case with the density of rods. This anatomical fact seems to be consistent with our result that both "E.Z.'s" at $10^{\circ}$ and $50^{\circ}$ are almost of the same value. Considering the anatomical basis mentioned above, we may explain our results as follows: At the middle periphery, the number of the stimulated rods will reach the maximum at a lower intensity of the stimulus (curve B in Fig. 3) and their predominance in number over the cones is so great that the "E.Z." determined by rods is not influenced even by an increase in the number of the stimulated cones. On the contrary, at the parafovea and at the extreme periphery, the "E.Z." depends on the relatively large increase in number of the stimulated cones with increasing intensities of the stimulus, because the ratio of the density of cones to that of rods at these "regions is far greater than that at the middle periphery. The minimum "E.Z." at about $30^{\circ}$ periphery of the dark-adapted eye may be due to stimulation of the largest number of rods. Thus, the question whether cones or rods predominate for the "E.Z." depends on the ratio of the densities of rods and cones stimulated.

The shortest" E. Z." determined by the cone function is obtained at the fovea, and that determined by the rod function at about $30^{\circ}$ periphery, the former being always shorter than the latter.

\section{Summary.}

"Empfindungszeit" ("E.Z.") for a wide range from the center to the periphery of the retina was measured by a modified Hazelhoff's method under various states of adaptation.

(1) It was proved that our method satisfied the necessary conditions for measuring the "E.Z." that an observer could make his gaze follow a fixation point travelling at a constant speed.

(2) The speed of the eye movement has no influence on the "E.Z." within the range of the speed from $10^{\circ}$ to $44^{\circ}$ per sec. in visual angle in the horizontal direction.

(3) At a constant intensity of a light stimulus, light adaptation causes an elongation of the "E.Z.", and the ratio of the "E.Z.'s" determined predominantly by the cone function at two different retinal parts remains constant irrespective of adaptation.

(4) Under dark adaptation, the value of the "E.Z." at a high intensity of the stimulus shows two minima at the fovea and about $30^{\circ}$ periphery and two maxima at about $10^{\circ}$ parafovea and the extreme periphery, while the value of the "E.Z." at a low intensity of the stimulus shows only a minimum at about $30^{\circ}$ periphery.

(5) The question whether cones or rods predominate for the "E.Z." seems to depend on the ratio of the densities of rods and cones of the retinal 
area stimulated. The shortest "E.Z.'s" determined by the cone function and by the rod function are obtained at the fovea and at about $30^{\circ}$ periphery respectively, and the former is shorter than the latter.

We wish to thank Prof. K. Motokawa for his advice and helpfulness.

\section{References.}

(1) Fröhlich, Fr. W., Die Empfindungszieit, G. Fischer, Jena, 1929.

(2) Von Kries, J., Naturwiss., 1923, 11, 461.

(3) Kronenberger, P., Arch. f. d. ges. Physiol., 1926, 211, 454.

(4) Hazelhoff, F.F. and Wiersma, H., Zeits. f. Psychol., 1924, 96, 171.

(5) 'Osterberg, G., Acta Ophth. Copenhagen, 1935, 13, Suppl. VI. 1. 